\title{
Indicadores de ciencia y tecnología en procesos de investigación, innovación y emprendimiento de la UNAN- MANAGUA
}

\author{
Indicators of science and technology in the research process, \\ innovation and entrepreneurship of the UNAN-MANAGUA
}

\author{
Juan de Dios Bonilla Anduray ${ }^{1}$ \\ Manuel Enrique Pedroza ${ }^{2}$
}

\section{RESUMEN}

Los Procesos de Investigación, Innovación y Emprendimiento están vinculados en las instituciones de educación superior cuya naturaleza está ligada al desarrollo humano y la producción de nuevos conocimientos. En la Universidad Nacional Autónoma de Nicaragua, UNAN-Managua, se desarrollan los procesos de CTI-ES, orientados por su Plan Estratégico Institucional 2015-2019. En este sentido, la implementación del Modelo $I+D+i$, permite la interacción de los procesos internos y externos a la institución en forma integral, holística, sistémica y antropocéntrica, facilitando el desarrollo del eje de Integración, Innovación y Modernización del Plan Estratégico Institucional. Por medio de indicadores de CTI-ES se visibiliza la producción de nuevos profesionales para fortalecer la vinculación universidad-empresa-sociedad. La universidad enfrenta el reto de trascendencia social y científica, al aportar para resolver los problemas sociales, tecnológicos, ecológicos e industriales, propios de la era del conocimiento, la información y los aprendizajes, por medio de la investigación, la innovación y los emprendimientos.

Palabras clave: indicadores de CTI-ES; Sistemas de Información Gerencial; Modelo I+D+i de la UNANManagua.

\begin{abstract}
The research process, innovation and entrepreneurship are linked in the higher education institutions whose nature is associated to human development and the production of new knowledge. At the National Autonomous University of Nicaragua, UNAN-Managua, CTI-ES processes are developed, guided by its Institutional Strategic Plan 2015-2019. In this sense, the implementation of the I + D + i Model allows the interaction of the internal and external processes to the institution in an integral, holistic, systemic and anthropocentric approach, facilitating the development of the alignment of Integration, Innovation and Modernization of the Institutional Strategic Plan. Through the indicators of CTI-ES the production of new professionals is visualized in order to strength the link between the universities, the enterprises and the society. The university faces the challenge of social and scientific transcendence, contributing to solve social, technological, ecological and industrial problems, typical of the era of knowledge, information and learning, through research, innovation and entrepreneurship.
\end{abstract}

Keywords: indicators of CTI-ES; Management System Information; Model I+D+I of UNAN-Managua.

DOI: https://doi.org/10.5377/multiensayos.v3i6.9694

Recibido: 05 de septiembre de 2017

Aceptado: 04 de diciembre de 2017

1 Docente UNAN-Managua, Managua. Estudiante del Programa de Doctorado en "Gestión y Calidad de la Investigación Científica". UNAN Managua. Correo electrónico: jbanduray@hotmail.com

2 Profesor Titular de la UNAN-Managua. Coordinador del Programa de Doctorado en "Gestión y Calidad de la Investigación Científica”. UNAN Managua. Correo electrónico: hppedroza@gmail.com 


\section{INTRODUCCIÓN}

La investigación está presente en el diario que hacer de los profesionales en la actualidad, es esencial para realizar un análisis del volumen de datos generados por el contexto global de las instituciones que cada vez más se encuentran más interconectadas e interdependientes entre sí. Al proceso formal de investigar se le conoce como investigación científica y tiene como característica el empleo del método científico y es la base del conocimiento actual de cada ciencia.

En Nicaragua, se han realizado estudios de investigación sobre el proceso de investigación científica, y han surgido resultados que la mayoría del conocimiento producto de investigaciones, es generado en las universidades. Se refleja también el desarrollo de las condiciones a nivel de gobierno sobre el interés en el tema de las investigaciones, prueba de ello es la inclusión en el Plan Nacional de Desarrollo Humano de proyectos de fortalecimiento al ente coordinador de la ciencia y la tecnología en el país, denominado CONICYT (Alemán Zeledón, Lezama Gaitán, Gómez, \& García, 2014).

En el año 2010, las Universidades del CNU desarrollaron un ejercicio tendiente a la construcción de indicadores de Ciencias y Tecnología en el ambiente universitario. De este esfuerzo se obtuvo un producto que en la práctica ha tenido muy pocos frutos, más allá de ser un documento base (Alemán Zeledón, Lezama Gaitán, Gómez, \& García, 2014).

En este contexto, se tiene conciencia de otro factor que impulsa el desarrollo de un país, como es el caso del proceso de innovación, algunos autores argumentan que es una necesidad vital para una organización. El proceso de innovación visto como generador de nuevas tecnologías, tiene varios caminos para desarrollarse, uno de ellos conocido como el "Fanel de la Innovación”, este consiste de una serie de pasos y de filtros para alcanzar la innovación, es decir, una secuencia de filtros sobre las ideas para alcanzar un producto o servicio (Muñoz, 2011).

La idea central de este método plantea una etapa inicial con el planteamiento del reto, este permite la generación de muchas ideas al respecto, a la lluvia de ideas se le aplica un primer filtro para desarrollar un concepto, con esto se descartan muchas ideas, partiendo del concepto, se utilizan técnicas para lograr establecer un plan de negocios, este documento ya refleja las posibilidades financieras y técnicas en el producto, para ello se aplican técnicas de análisis con posibilidades de obtener un proyecto y gestionarlo (Muñoz, 2011).

La UNAN-Managua en su plan estratégico plantea la innovación como eje transversal y presente en cada etapa del desarrollo curricular para la formación de habilidades y capacidades de los futuros profesionales estando inmerso en el modelo educativo. Por tal razón, se ha creado una estructura organizacional que atiende el proceso de innovación y su desarrollo, además de fomentar las iniciativas de proyectos por medio de un programa de fondos concursables (UNAN-Managua, 2015). 
El plan estratégico es un documento claro, conciso y operacional que fortalece el posicionamiento de la UNAN-Managua en el periodo 2015-2019, su concepción participativa orienta a la mejora continua a partir de la institucionalización de una cultura de planificación y evaluación, del fortalecimiento de la docencia, la investigación, la innovación y emprendimiento; de la implementación de procesos administrativos agiles y eficientes con articulación e integración efectiva en los espacios de intercambio con el estado, la empresa privada y la sociedad en general (UNAN-Managua, 2015).

La función investigación en el plan estratégico de la UNAN-Managua, se establece como la creación de conocimientos, es una función sustantiva de la Universidad, que debe ser aprovechada para contribuir a la transformación de la sociedad, su mejoramiento y adaptación a las nuevas tecnologías de acuerdo con las demandas y necesidades del desarrollo humano sostenible del país (UNAN-Managua, 2015).

Derivado del proceso de innovación en la UNAN-Managua surge el fomento al emprendimiento, teniendo como fuente los proyectos de innovación ganadores de los fondos 2015.

Por otra parte, el estado de Nicaragua plantea en el plan de Ciencia, Tecnología e Innovación 2010-2013, el desarrollo de un sistema de indicadores de ciencia, tecnología e innovación para fortalecer el proceso de diseño y ejecución de políticas de Ciencia, Tecnología e Innovación y la toma de decisiones de los actores del Sistema Nacional de Investigadores de Nicaragua (Alemán Zeledón, Lezama Gaitán, Gómez, \& García, 2014), como resultado de esta iniciativa se lograron algunos avances en este campo, sin embargo no se automatizó el proceso y como consecuencia sigue presentándose un sub registro de la producción intelectual y técnica del país.

\section{DESARROLLO}

\section{Contexto Institucional de los Procesos en estudio}

Un primer aporte del estudio corresponde al análisis cualitativo de la condición legal de los procesos de investigación, innovación y emprendimiento que tienen en la UNAN Managua, razón por la cual se describe a continuación el estado antiguo de los procesos y lo establecido actualmente en el plan estratégico institucional para el periodo 2015 - 2019.

El proceso de Investigación es el más desarrollado, posee normativas que controlan el desarrollo de las actividades generales de la UNAN-Managua como son la JUDC y los congresos científicos docentes.

Respecto a los procesos de innovación y emprendimiento en estudio, se tiene que en un principio (año 2011), se encontraban ubicados en la dirección de extensión universitaria, por tal motivo es relevante señalar lo que refleja el artículo 10 de la política de extensión. 
En mayo del 2011 se crea la Vice rectoría de Investigación, Posgrado y Extensión Universitaria (VRIPEU) esto para integrar los procesos de investigación en el grado y posgrado, también lo de extensión universitaria. Dentro de la nueva estructura en VRIPEU. El 27 de noviembre del 2011, el Dr. Manuel Enrique Pedroza, siendo el Director del Área de Investigación de la UNAN-Managua, presentó por primera vez ante la Comisión de Investigación, Comisión de Posgrado y Comisión de Extensión, (las tres comisiones sesionando en conjunto), presentó el documento del Modelo $I+D+i$ de la UNAN-Managua, el cual fue aprobado por unanimidad con pequeñas recomendaciones.

En su sección III, articulo 10 la política de extensión establece que se "Tiene como finalidad, la creación de una cultura de emprendimiento e innovación en docentes y estudiantes de la UNANManagua (UNAN-Managua. Dirección de Extensión Universitaria, 2012):

- Se promoverán amplios espacios de capacitación a docentes y estudiante en los temas de innovación y emprendimiento.

- Se formará la creación de una cultura emprendedora entre docentes y estudiantes, a través de la asignación de recursos, premios e incentivos a toda actividad, que conlleva a la creación de productos, bienes y servicios, que mejoren la calidad educativa de la institución y/o la competitividad económica del país;

- A través de la oficina de Enlace y Promotoría de la Dirección de Extensión Universitaria, se dará asistencia técnica en los temas de patentes y propiedad intelectual.

- Se fomentará la participación activa de docentes y estudiantes de emprendimiento e innovación;

- Se fomentará el reconocimiento institucional a innovadores y emprendedores destacados."

En julio de 2014, se crea la Vice rectoría de Investigación y se separan las funciones de investigación y extensión. Dentro de esta Vice rectoría se ubica la Dirección de Investigación de Grado y la Dirección de Investigación de Posgrado. Dentro de la Dirección de Investigación de Grado, el Dr. Manuel Enrique Pedroza, siendo su Director, creo el Área de Innovación y Emprendimiento, el Área de Gestión del Conocimiento, el Área de Propiedad Intelectual y el Área de Publicaciones Científicas. De esta forma se creó una Innovación Organizacional de la Dirección de Investigación, la cual está fundamentada en el Modelo I+D+i de la UNAN-Managua, con visión de largo plazo y sentido de nación, que fue presentada en el Congreso Mesoamericano de Investigación en Chiapas, México, en octubre 2016 (Pedroza M. E., 2015). La estructura organizativa actual de la Dirección de Investigación de Grado se presenta en la figura 1. 

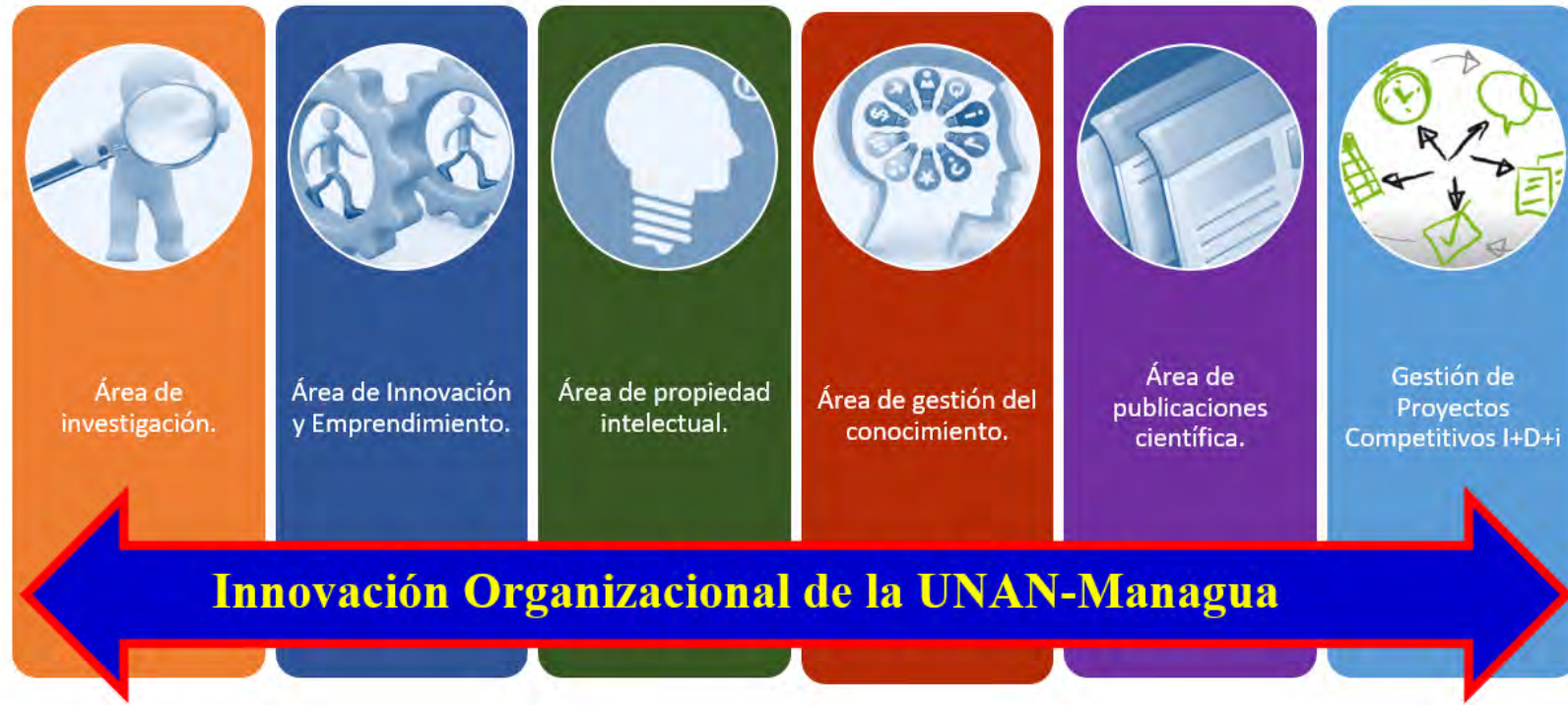

\section{Innovación Organizacional de la UNAN-Managua}

Figura 1. Estructura organizativa de la Dirección de Investigación de Grado, 2014-2018. Fuente Dirección de Investigación de Grado.

Basada en el Modelo I+D+i de la UNAN-Managua desde el año 2011, esta nueva organización institucional de la investigación científica de la UNAN-Managua fue implementada en el año 2014, vino a cambiar la concepción y actuación tradicional mono disciplinaria, que existía en torno al quehacer de la investigación científica de la UNAN-Managua (descriptiva principalmente) y la impulsó más allá a niveles de integración institucional con otros procesos científico tecnológicos (CTI), con los cuales la investigación científica mantiene vínculos indivisibles. Se inició así un cambio muy importante hacia "una nueva cultura institucional de investigación científica" orientada hacia resultados con calidad y pertinencia.

Este cambio hacia "una nueva cultura institucional de investigación cientifica" ha venido a promover en la UNAN-Managua el eje de Integración, Innovación y Modernización del Plan Estratégico Institucional 2015-2019, incidiendo en la formación profesional de los estudiantes $y$ docentes, mediante el desarrollo de procesos integrados de investigación-innovaciónemprendimiento, con vinculaciones indivisibles con el Área de gestión del conocimiento, el Área de publicaciones cientifica, el Área de propiedad intelectual y el Área de gestión de programas $y$ proyectos competitivos $I+D+i$, que desde el inicio constituyó parte integral de la propuesta de Innovación Organizacional, solamente que esta área fue asignada como una función de la Dirección de Investigación de Posgrado.

Esta "nueva cultura institucional de investigación científica", es la que garantiza el fortalecimiento institucional de los procesos de extensión universitaria y proyección social, tanto a lo interno como a lo externo de la universidad, lo cual ha tenido impactos positivos que han fortalecido la propia institucionalidad de la universidad, transformando positivamente la organización, normativa y 
procedimientos de la JUDC desde el año 2012 (Normativa del 2012 y 2014), fortaleciendo la investigación como eje transversal del currículo, creando las Líneas de Investigación de Grado de la UNAN-Managua en el año 2012 y actualizándolas posteriormente en el año 2016, promoviendo fuertemente la divulgación científica-técnica a nivel de cada facultad para tener su propia revista electrónica, creando programas de maestría y doctorado en investigación científica (MEDINV en FAREM Carazo y DOGCINV en FAREM Estelí), promoviendo el modelo educativo, la misión y visión de la UNAN-Managua, promoviendo la integración institucional a nivel de grado y posgrado, promoviendo políticas de investigación, innovación y emprendimiento que faciliten y consoliden la integración de estos procesos y que simultáneamente promuevan la integración institucional de las cinco funciones vitales de la universidad: Educación, Investigación, Extensión, Internalización y Gestión Administrativa.

A partir de esta nueva organización institucional, se ha venido impulsando y apoyando todas las iniciativas que conllevan al desarrollo de los procesos de innovación que, actualmente tienen tres grandes procesos institucionales de UNAN-Managua: (a) El "Taller de Innovación de FAREM Esteli', impulsado con el apoyo del Dr. Paul Lane de Grand Valley University, realizado anualmente con una amplia participación de estudiantes y docentes de las diez facultades de la UNANManagua; (b) El "Fondo de Innovación de la UNAN-Managua”, que promueve diversos proyectos de innovación con estudiantes de grado y docentes basado en criterios de calidad y pertinencia, orientado su quehacer institucional por la "Normativa para la selección y ejecución de proyectos de innovación de la UNAN-Managua, mediante el mecanismo de Fondos Concursables” y (c) participación en el "Concurso Nacional de Innovación promovido anualmente por el CONICYT", en el que participan diferentes proyectos de innovación realizados por estudiantes de grado y docentes, (UNAN-Managua. Dirección de Investigación, 2015).

Las bases de la "Normativa para la selección y ejecución de proyectos de innovación de la UNANManagua, mediante el mecanismo de Fondos Concursables", que deberán observarse por su relevancia en el estudio, son las siguientes:

1. Participan todos los estudiantes activos de segundo, tercero y cuarto año de las carreras de la UNAN - Managua.

2. Participan todos los docentes de UNAN Managua como tutores y proyectistas.

3. Se considerarán principalmente los proyectos de innovación con enfoque multi -inter y trans disciplinarios.

4. Los proyectos que podrán participar en dicha convocatoria serán los siguientes:

4.1. Proyectos originarios del Taller de Innovación realizado en la Ciudad de Estelí en los meses de Mayo de cada año.

4.2. Proyectos de Innovación que fueron presentados en la JUDC realizada en la UNAN Managua, correspondiente al año anterior de la convocatoria vigente.

4.3. Proyectos de Innovación presentados en las Ferias Territoriales y Tecnológicas realizada por el CNU y CONICYT, correspondiente al año anterior de la convocatoria vigente. 
5. Los proyectos de innovación deberán responder al menos a una línea de innovación establecida en la presente convocatoria UNAN - Managua.

6. Los proyectos de innovación tendrán que responder a una problemática real y basadas en las áreas priorizadas (Punto VI de la presente normativa), de pertinencia con el contexto social, productivo y económico de Nicaragua.

7. Cada proyecto estará formado por un equipo de trabajo constituido por un mínimo de dos y un máximo cinco integrantes, y con dos disciplinas diferentes (se exceptúa de esto último a los proyectos de estudiantes de UNICAM, ya que su formación es esencialmente Multidisciplinaria). Por tanto, se deberá designar un coordinador, el cual debe demostrar responsabilidad y disciplina.

8. Los proyectos presentados por los estudiantes, deberán tener de uno a dos docentes que funcionaran como tutores de todo el proceso de innovación, para lo cual el proyecto lo trabajaran de forma conjunta.

9. Los estudiantes innovadores deberán presentar como soporte a su solicitud, una carta de un tutor, en donde este se compromete a garantizar el desarrollo eficiente y transparente del proyecto de innovación.

10. Para el caso de los docentes deberá presentar una carta de aval de su respectivo Decano (a) o Director (a), según sea el caso.

11. El periodo de ejecución del proyecto será de tres meses, contándose a partir de la firma del contrato de adjudicación de los fondos.

12. Los fondos tienen que ejecutarse y rendir cuenta de los mismos a más tardar el 30 de octubre del año en curso.

13. El presupuesto del proyecto debe estar acorde al cronograma de actividades del proyecto. Cada gasto debe presentar su justificación. Los proyectos que presenten mediante gastos globales no serán aceptados.

14. El rubro de viáticos y transporte no podrá ser más del $20 \%$ con respecto al monto global del presupuesto del proyecto de innovación presentado.

15. El rubro de compra de equipos o medios, contrataciones de servicios profesionales y otros gastos vinculados a la Construcción del Prototipo y Modelos Funcional, no podrá ser más del $70 \%$ con respecto al monto global del presupuesto del proyecto de innovación presentado.

16. El 10\% restante será utilizado para la visibilización / divulgación de los procesos de innovación, durante el periodo de ejecución del prototipo.

Es evidente que estas disposiciones facilitan e impulsan el desarrollo de los procesos de investigación e innovación en la UNAN-Managua. Sin embargo, con este mayor desarrollo se requiere la medición correspondiente y para ello es necesario un sistema de registro y control automatizado de Indicadores de CTI (innovación), para monitorear, evaluar y controlar la calidad y pertinencia de estos procesos.

En esta nueva organización, uno de los actores importantes para el desarrollo de los procesos de innovación y emprendimiento, corresponde a la Comisión de Innovación a nivel facultativo y cuyo 
coordinador debe realizar las acciones orientadas según la normativa publicada en la página web de la UNAN-Managua (UNAN-Managua. Comisión Facultativa POLISAL, 2015).

El reglamento interno de la UNAN-Managua establece en su apartado de Naturaleza de la Unidad de Investigación e Innovación en la Facultad, lo que se detalla a continuación:

"La unidad de investigación e innovación de las facultades es la instancia de la dirección de investigación que regula, promueve y divulga la organización de procesos de investigación, desarrollo e innovación, mediante la constitución de equipos de trabajos multi, ínter y trans disciplinario. Tiene como función fundamental contribuir al desarrollo sostenible del país, dando respuesta a los problemas que demanda la sociedad, considerando las prioridades establecidas por el Plan Estratégico Institucional de la UNAN -Managua 2015-2019 y el Plan Nacional de Desarrollo Humano (PNDH)".

La unidad de investigación e innovación está en correspondencia con la misión y visión institucional de la UNAN -Managua según áreas del conocimiento de sus Facultades y POLISAL y departamentos docentes.

Así mismo, el reglamento (UNAN-Managua. Dirección de Investigación, 2015) establece las funciones del coordinador de innovación facultativo en el artículo 7 y establece lo siguiente:

a. Formar parte y concurrir a las sesiones y/o reuniones que planifique el Vicerrectorado y la Dirección de Investigación de Grado y Postgrado, con voz y voto.

b. Participar en las actividades o eventos científicos que planifique el Vicerrectorado y la Dirección de Investigación de Grado y Postgrado de la UNAN -Managua a nivel interno y externo de la Institución.

c. Participar en la elaboración de Reglamentos y Normativas relacionadas con la Investigación y la Innovación.

d. Participar en comisiones ad-hoc orientadas por el Vicerrectorado, Dirección de Investigación de Grado y Postgrado y el Decanato.

e. Proponer Proyectos de Investigación con enfoque ínter, multi y trans disciplinario a nivel de la Facultad, POLISAL e Institucional.

f. Promover Proyectos de Innovación en el grado y postgrado a nivel de la Facultad, POLISAL e Institucional.

g. Proponer al Consejo Universitario los proyectos de investigación e innovación que deban ser financiadas por la Institución.

h. Elaborar, monitorear y evaluar el POA, informes trimestrales, e informes finales de acuerdo a lo especificado por el Vicerrectorado por medio de las direcciones de Investigación de Grado y Postgrado.

Por otra parte, el proceso de emprendimiento se deriva de los resultados y desarrollo de la innovación presentando un inicio en el documento base presentado por la Dirección de Investigación de Grado. 
La Dirección de Investigación de la UNAN-Managua ha creado desde noviembre 2011, el Modelo $I+D+i$ de la UNAN-Managua. El Modelo $\mathrm{I}+\mathrm{D}+\mathrm{i}$, es un sistema en sí mismo y como tal expresa un conjunto de conexiones, relaciones e interacciones de actores y factores, que conllevan a la complementariedad y sinergia de cada uno de los componentes de este sistema. El Modelo I+D $+\mathrm{i}$ de la UNAN-Managua, promueve cuatro etapas (Pedroza M. E., 2015)

En una primera etapa, se deben iniciar los procesos de investigación con la observación y definición del problema objeto de estudio, esto es orientado a la búsqueda de solución a los problemas reales, propios de las demandas y necesidades de los actores locales en los territorios. De esta forma el modelo $\mathrm{I}+\mathrm{D}+\mathrm{i}$ se inicia abordando problemas en la realidad y basado en los conocimientos existentes desde el nivel universitario.

Una segunda etapa, es la evolución natural del nuevo conocimiento generado en la investigación, hasta transitar hacia el desarrollo de nuevos prototipos. En esta segunda etapa se incluye necesariamente la Gestión del Conocimiento y el desarrollo de Redes de Actores, que facilitan tanto la captura de nuevas tecnologías como la adopción, adaptación y aplicación de los nuevos prototipos.

La tercera etapa, es impulsar la gerencia de la investigación científica, desde la identificación, formulación, monitoreo y evaluación de Proyectos Competitivos $\mathrm{I}+\mathrm{D}+\mathrm{i}$, hasta lograr el registro de patentes, propiedad intelectual y marca, que permitan la implementación posterior de programas, proyectos y planes de negocios basados en las nuevas tecnologías.

En una cuarta etapa, se deben implementar las iniciativas de nuevos emprendimientos, basados en los planes de negocios, programas y proyectos de las nuevas tecnologías que se concretizaron en los proyectos competitivos $\mathrm{I}+\mathrm{D}+\mathrm{i}$ de la tercera etapa, hasta llevarlos al desarrollo de nuevas empresas.

La implementación de las cuatro etapas, constituye la evolución natural a seguir, para hacer realidad la integración efectiva de los procesos de Investigación, Innovación y Emprendimientos. Estas cuatro etapas promueven la complementariedad y sinergia de cada una de las instancias de la UNAN-Managua, que tengan por definición institucional la responsabilidad de realizar cada uno de estos procesos (Pedroza M. E., 2015).

En el año 2015, se propone el documento Propuesta de una Oficina de Incubación de Empresas en UNAN-Managua. Esta propuesta plantea tres etapas, la Pre incubación, Incubación y Post-incubación de empresas. Esta propuesta facilitará la preparación de negocios de los emprendedores facilitando la creación de nuevas empresas mediante servicios integrales de asesoría, acompañamiento y capacitación durante la etapa de creación y maduración como negocio (UNAN-Managua. Dirección de Extensión Universitaria, 2012). 
El documento también plantea un catálogo de servicios en las diferentes etapas que forman el emprendimiento, a continuación se especifican por etapa (UNAN-Managua, Dirección de Investigación, 2014).

\section{Pre- Incubación}

1. Capacitación en diseños de producto o servicios.

2. Capacitación en la elaboración de planes de negocios.

3. Capacitación en paquetes estadísticos.

4. Acompañamiento y monitoreo en el diseño del plan de negocio.

5. Capacitación y acompañamiento en el registro de marca y patentes (mediante la oficina CATI).

6. Gestiones de colaboración con Facultades, Direcciones, Centros, Institutos y Laboratorios especializados de UNAN Managua.

7. Vinculaciones a nivel externo con instituciones públicas y privadas.

8. Promoción de participación en talleres, ferias, foros, competencias, entrevistas, conferencias, congresos y capacitaciones.

9. Proyectar a los nuevos emprendedores de los medios de comunicación.

\section{Incubación}

1. Apoyo en la gestión de fuentes de financiamiento inicial (capital semilla) en vinculación con distintas unidades de UNAN Managua.

2. Acompañamiento y monitoreo en el desarrollo inicial de la empresa (tiempo de duración: 1 año).

\section{Post- incubación de empresas}

1. Monitoreo a las empresas incubadas.

2. Asesoramiento a las empresas para la mejoras de los productos, servicios o procesos.

En este momento la UNAN-Managua se encuentra en el proceso de Pre incubación de empresas, cuyo objetivo es preparar los mejores proyectos de innovación seleccionados, mediante la elaboración de planes de negocios. El periodo de esta etapa debe estar comprendido en lapso de siete meses.

El área de innovación y emprendimiento de la .UNAN Managua con apoyo de la sub comisión de emprendimiento están a cargo de la organización de dichos talleres así como las asesorías, acompañamientos y vinculaciones para lograr el éxito de dicho programa. Los talleres serán los siguientes:

1. Taller sobre plan de marketing.

Proceso de acompañamiento y monitoreo al plan de marketing. 
2. Taller sobre plan de producción.

Proceso de acompañamiento y monitoreo del plan de producción.

3. Taller sobre Plan de organización y gestión.

Proceso de acompañamiento y monitoreo al plan de organización y gestión.

4. Taller sobre plan financiero.

Proceso de acompañamiento y monitoreo al plan de organización y gestión.

El acompañamiento y asesoría puede ser de manera presencial o virtual. Para cada taller se seleccionarán especialistas de dichas temáticas a nivel interno o externo.

Se gestiona el apoyo de los Centros y Laboratorios de Investigación de UNAN Managua. Después de la elaboración de los planes de negocios los estudiantes harán una presentación con la comisión de innovación de UNAN Managua y se les hará entrega de un diploma de participación como estímulo. Los mejores proyectos serán llevados al proceso de incubación de empresas.

\section{Logros Relevantes en los Procesos de Investigación, Innovación y Emprendimiento de la UNAN-Managua al año 2016}

Los logros alcanzados en los procesos de Investigación, Innovación y Emprendimiento en la UNAN-Managua al año 2015, se destacan en el cumplimiento de algunos indicadores de CTI, los que se referencian a partir del Informe de Logros 2015 (UNAN-Managua. Dirección de Investigación, 2015).

El impacto de los procesos de Investigación, Innovación y Emprendimiento de la UNAN-Managua, se demuestra en los resultados globales alcanzados en el año 2015 evidenciados en el Informe Anual de la XXXIV JUDC 2015, en el que se demostró una amplia y creciente participación de la comunidad universitaria de la UNAN-Managua: en la XXXIV JUDC 2015 se presentaron 1347 trabajos en total, con una participación estudiantil de 3127 estudiantes expositores y un apoyo activo de 555 docentes que trabajaron como tutores de los diferentes trabajos presentados. Esto significa que con respecto al año 2011, se alcanzó un cumplimiento del 180,32 \% en temas presentados, un 163,28 \% estudiantes expositores y un $165 \%$ en tutores de la JUDC. Estos son indicadores de CTI que demuestran claramente un impacto positivo de una creciente productividad científica de la UNAN-Managua.

Esta creciente productividad científica, alcanzada a través de la JUDC 2015, se distribuye de la siguiente manera según el tipo de trabajo presentado: 536 protocolos de investigación, 283 informes de investigación científica, 172 ensayos, 71 evaluaciones de software de aplicación o páginas web, 60 proyectos de innovación, 51 proyectos de inversión y desarrollo, 34 artículos científicos, 32 investigaciones participativas, 25 investigaciones de mercado, 23 informes 
preliminares, 22 pre defensas de trabajos monográficos, 11 diagnósticos, 11 trabajos sobre casos clínicos, 10 investigaciones sobre procesos de enfermería, 5 informes de prácticas y finalmente 1 trabajo sobre sistematización de procesos. El impacto positivo de esta creciente productividad científica se sintetiza en: más Protocolos de Investigación, más Informes de Investigación, más Proyectos de Innovación, más Ensayos, más Proyectos de I \& D, más Aplicaciones TIC (UNANManagua. Dirección de Investigación, 2015).

Esta tendencia de creciente productividad científica, a través de la JUDC, se demostró también en la XXXV JUDC del año 2016. En el Informe de la XXXV JUDC del año 2016, se demuestra que, en el año 2016, se presentaron 1405 trabajos en total, con una participación estudiantil de 3437 estudiantes expositores y un apoyo activo de 715 docentes que trabajaron como tutores de los diferentes trabajos presentados (UNAN-Managua. Dirección de Investigación, 2016). Esto significa un impacto institucional muy importante con respecto al año 2011, se alcanzó un cumplimiento del $188,09 \%$ en temas presentados, un $179,48 \%$ estudiantes expositores y un $212.17 \%$ en tutores de la JUDC. Estos son indicadores de CTI que demuestran claramente un impacto positivo de una creciente productividad científica de la UNAN-Managua.

En lo que respecta al proceso de Innovación, se realizaron dos eventos de mucho impacto: Un foro Multidisciplinario de Innovación y Emprendimiento, organizado y realizado por la FAREM Estelí y un Foro de Innovación en Salud, organizado y realizado por la Facultad de Ciencias Médicas y el POLISAL. Además, se destaca el fortalecimiento de la Red de Jóvenes y Docentes Investigadores, Innovadores y Emprendedores de UNAN-Managua.

En el área de CTI, se destaca el congreso de Informática Educativa, implementando 4 mesas técnicas, 4 ponencias y un panelista.

Con los Fondos de Innovación 2015 se financiaron 15 proyectos, resultando 15 prototipos funcionales, de los cuales se seleccionaron 6 proyectos para iniciar el proceso de pre incubación de empresas, 3 de ellos están en mejoramiento de diseño, 2 en investigación de mercado y 1 en plan de producción.

En el contexto territorial, se destacan los procesos participativos de las expociencias territoriales la UNAN-Managua presentó un total de 183 proyectos.

En el contexto nacional, se participó en los fondos de apoyo a la innovación del CONICYT, donde se alcanzó el Primer lugar en la categoría de industria por el laboratorio de Bio-Tecnología. A nivel del Consejo Nacional de Universidades (CNU), se destaca la participación de la UNANManagua en el $X V$ Encuentro de Investigación, Postgrado y Extensión Universitaria, realizado el 5 de septiembre 2015 en la UNAN-León bajo el lema "Retos y desafíos para la Investigación, Posgrado, y Extensión Universitaria", en donde se presentó como la conferencia central del XV Encuentro, el libro del Modelo de Gestión de la Investigación: Modelo I+D+i de la UNAN- 
Managua. Por tota parte, desde su propio inicio en noviembre 2014 hasta la fecha, la UNANManagua se ha destacado por su plena participación y apoyo institucional al INTA en el esfuerzo nacional de la instalación y desarrollo del Sistema Nicaragüense de Investigación e Innovación Agropecuaria -SNIA- (UNAN-Managua. Dirección de Investigación, 2015).

Es de gran relevancia destacar el indicador de publicaciones científicas de la UNAN-Managua al año 2015, en el cual existen avances muy importantes:

- 14 Revistas en Línea, en Plataforma OJS.

- De ellas 9 Revistas Indexadas.

- Dos cursos de Capacitación en OJS solo en el año 2015.

- Tres cursos de Capacitación en Redacción Científico-Técnica.

- Tres Revistas Arbitradas.

- 6 Nuevas Revistas en Proceso de Edición.

- 1 Encuentro Anual de Editores de Revistas.

Por otra parte, el impacto positivo de las investigaciones de los docentes fue evidenciado en el XIX Congreso Científico de la UNAN-Managua 2015, que incluyó 10 congresos científicos, realizados en cada una de las facultades de la UNAN-Managua. Los congresos realizados en la ciudad de Managua, estaban distribuidos en las diferentes Facultades que forman los recintos universitarios Rubén Darío y Carlos Fonseca Amador.

El XIX Congreso Científico de la UNAN-Managua 2015, significó otro indicador de CTI de gran impacto en la tendencia creciente de productividad científica de la UNAN-Managua. Como medición los logros alcanzados en esta jornada científica de los docentes, se resumen en el cumplimiento de: un 103,27 \% de las mesas del congreso programadas, un 133,33\% de las conferencias magistrales programadas, un $109 \%$ de las ponencias programadas, un $97 \%$ de los panelistas programados, confirmando una tendencia creciente de productividad científica de la UNAN-Managua, sin precedentes. Por tanto, este XIX Congreso Científico 2015 de la UNANManagua, marcó un hito en la historia de la vida institucional. En el cuadro 1, se presenta el cumplimiento alcanzado (UNAN-Managua. Dirección de Investigación, 2015)..

Cuadro 1. Cumplimiento XIX Congreso Científico de la UNAN-Managua. 2015.

\begin{tabular}{|l|c|c|c|c|c|c|c|c|}
\hline $\begin{array}{l}\text { Facultad/ } \\
\text { Centro }\end{array}$ & Mesas & $\begin{array}{l}\text { Cumpli- } \\
\text { miento }\end{array}$ & Magistrales & $\begin{array}{l}\text { Cumpli- } \\
\text { miento }\end{array}$ & Ponencias & $\begin{array}{l}\text { Cumpli- } \\
\text { miento }\end{array}$ & $\begin{array}{l}\text { Pa- } \\
\text { nelistas }\end{array}$ & $\begin{array}{l}\text { Cumpli- } \\
\text { miento }\end{array}$ \\
\hline $\begin{array}{l}\text { Facultad de } \\
\text { Humanidades }\end{array}$ & 11 & 17 & 1 & 1 & 70 & 77 & 6 & 5 \\
\hline $\begin{array}{l}\text { Facultad de } \\
\text { Ciencias } \\
\text { Médicas }\end{array}$ & 4 & 2 & 4 & 7 & 30 & 31 & 4 & 4 \\
\hline POLISAL & 2 & 2 & 1 & 2 & 18 & 18 & 0 & 0 \\
\hline $\begin{array}{l}\text { Facultad CC } \\
\text { e Ing }\end{array}$ & 5 & 5 & 2 & 3 & 40 & 65 & 6 & 4 \\
\hline
\end{tabular}




\begin{tabular}{|l|c|c|c|c|c|c|c|c|}
\hline $\begin{array}{l}\text { Facultad de } \\
\text { CC EE }\end{array}$ & 3 & 1 & 3 & 3 & 33 & 11 & 0 & 0 \\
\hline $\begin{array}{l}\text { FAREM- } \\
\text { Estelí }\end{array}$ & 10 & 10 & 3 & 2 & 33 & 34 & 3 & 0 \\
\hline $\begin{array}{l}\text { FAREM- } \\
\text { Chontales }\end{array}$ & 5 & 5 & 4 & 10 & 25 & 43 & 3 & 6 \\
\hline $\begin{array}{l}\text { FAREM- } \\
\text { Matagalpa }\end{array}$ & 3 & 3 & 1 & 1 & 30 & 35 & 0 & 0 \\
\hline $\begin{array}{l}\text { FAREM- } \\
\text { Carazo }\end{array}$ & 3 & 3 & 4 & 4 & 12 & 12 & 3 & 3 \\
\hline IGG/CIGEO & 1 & 1 & 3 & 3 & 14 & 13 & 1 & 3 \\
\hline $\begin{array}{l}\text { Facultad de } \\
\text { Educación }\end{array}$ & 5 & 5 & 1 & 1 & 40 & 40 & 4 & 4 \\
\hline $\begin{array}{l}\text { Congreso de } \\
\text { Informática } \\
\text { educativa }\end{array}$ & 4 & 4 & 0 & 0 & 4 & 0 & 1 & 1 \\
\hline $\begin{array}{l}\text { I Foro } \\
\text { Innovación } \\
\text { en Salud }\end{array}$ & 5 & 5 & 3 & 3 & 3 & 3 & 0 & 0 \\
\hline TOTAL & 61 & 63 & 30 & 40 & 352 & 382 & 31 & 30 \\
\hline
\end{tabular}

Fuente: Informe de Logros 2015 de la DIRINVES.

Situación de los Indicadores de Ciencia, Tecnología e Innovación

El Dr. Manuel Enrique Pedroza, declara en su mensaje del primer aniversario (7 de julio 2017) del Programa de doctorado en Gestión y Calidad de la Investigación Científica (DOGCINV) que: "para ser exitosos y exitosas en nuestras tareas, debemos tener una actitud positiva y socioconstructivista frente a la vida, actitud en esencia de lucha, esfuerzos y sacrificios, siendo proactivos y propositivos, con disciplina, gerencia de tiempo, identidad y compromiso para nuestra Universidad UNAN-Managua, llenos de convicción en que nuestro trabajo si rendirá buenos frutos a corto, mediano o largo plazo. Seguramente esos principios nos pondrán en el camino de la productividad y la calidad, fortaleciendo los procesos de educación, investigacióninnovación-emprendimiento, guiados por el modelo $I+D+i$ de UNAN-Managua, el cual en pocas palabras nos mantendrá en el camino de CTI-ES como un medio fundamental para el desarrollo humano sostenible de la familia nicaragüense”, (Pedroza M. E., 2017).

Cabe destacar que el modelo $\mathrm{I}+\mathrm{D}+\mathrm{i}$ de la UNAN-Managua es una visión panorámica y completa del camino a seguir por las diferentes instituciones que se dedican al desarrollo de CTI-ES en Nicaragua, muy acorde con el propósito del Programa Aprender Emprender Prosperar que impulsa el estado de Nicaragua para el bienestar e integración de los sub sistemas de educación.

En el contexto nacional, actualmente la situación de los Indicadores de CTI, se concretiza en la propuesta de (Alemán Zeledón, Lezama Gaitán, Gómez, \& García, 2014), quienes plantean los 
principales indicadores a nivel del CNU e instituciones de CTI de Nicaragua, tales indicadores fundamentan el presente estudio:

\begin{tabular}{|c|c|c|c|}
\hline Dimensión & Indicador & Dimensión & Indicador \\
\hline \multirow[t]{7}{*}{$\begin{array}{l}\text { Recursos humanos } \\
\text { dedicados a ciencia, } \\
\text { tecnología e } \\
\text { innovación }\end{array}$} & $\begin{array}{l}\text { Número de personas } \\
\text { dedicadas a tiempo } \\
\text { completo a ciencia, } \\
\text { tecnología e innovación, } \\
\text { por sexo y tipo de } \\
\text { función. }\end{array}$ & \multirow[t]{2}{*}{$\begin{array}{l}\text { Programas y proyectos } \\
\text { de ciencia, tecnología } \\
\text { e innovación por área } \\
\text { científica }\end{array}$} & $\begin{array}{l}\text { Número de programas } \\
\text { de ciencia, tecnología e } \\
\text { innovación en proceso, } \\
\text { por área científica, para el } \\
\text { año } 2017\end{array}$ \\
\hline & $\begin{array}{l}\text { Número de investigadores } \\
\text { de tiempo parcial } \\
\text { dedicado a ciencia, } \\
\text { tecnología e innovación } \\
\text { por sexo }\end{array}$ & & $\begin{array}{l}\text { Número de programas } \\
\text { de ciencia, tecnología e } \\
\text { innovación concluidos, } \\
\text { por área científica, para el } \\
\text { año } 2017\end{array}$ \\
\hline & $\begin{array}{l}\text { Número de estudiantes } \\
\text { de doctorado de tiempo } \\
\text { parcial dedicado a } \\
\text { ciencia, tecnología e } \\
\text { innovación por sexo. }\end{array}$ & $\begin{array}{l}\text { Alianzas estratégicas } \\
\text { en las que participa } \\
\text { la institución para } \\
\text { desarrollar ciencia, } \\
\text { tecnología e innovación }\end{array}$ & $\begin{array}{l}\text { Número de convenios y } \\
\text { participación en redes de } \\
\text { colaboración de ciencia, } \\
\text { tecnología e innovación, } \\
\text { por área científica. }\end{array}$ \\
\hline & $\begin{array}{l}\text { Número de estudiantes } \\
\text { de maestría académica de } \\
\text { tiempo parcial dedicado } \\
\text { a ciencia, tecnología e } \\
\text { innovación por sexo }\end{array}$ & $\begin{array}{l}\text { Infraestructura para } \\
\text { desarrollar ciencia, } \\
\text { tecnología e innovación }\end{array}$ & $\begin{array}{l}\text { No. de laboratorios que } \\
\text { disponen de equipamiento } \\
\text { básico y especializado por } \\
\text { área científica. }\end{array}$ \\
\hline & $\begin{array}{l}\text { Número de personal } \\
\text { técnico y de apoyo } \\
\text { de tiempo completo } \\
\text { dedicado a ciencia, } \\
\text { tecnología e innovación } \\
\text { por sexo y función. }\end{array}$ & \multirow[t]{3}{*}{ Innovaciones } & $\begin{array}{l}\text { Número de innovaciones } \\
\text { de productos (bienes) }\end{array}$ \\
\hline & $\begin{array}{l}\text { Número de técnicos de } \\
\text { laboratorio de tiempo } \\
\text { parcial dedicado a } \\
\text { ciencia, tecnología e } \\
\text { innovación por sexo }\end{array}$ & & $\begin{array}{l}\text { Número innovaciones de } \\
\text { productos (servicios) }\end{array}$ \\
\hline & $\begin{array}{l}\text { Número de personal de } \\
\text { apoyo de tiempo parcial } \\
\text { dedicado a ciencia, } \\
\text { tecnología e innovación } \\
\text { por sexo }\end{array}$ & & $\begin{array}{l}\text { Número de innovaciones } \\
\text { de procesos }\end{array}$ \\
\hline
\end{tabular}




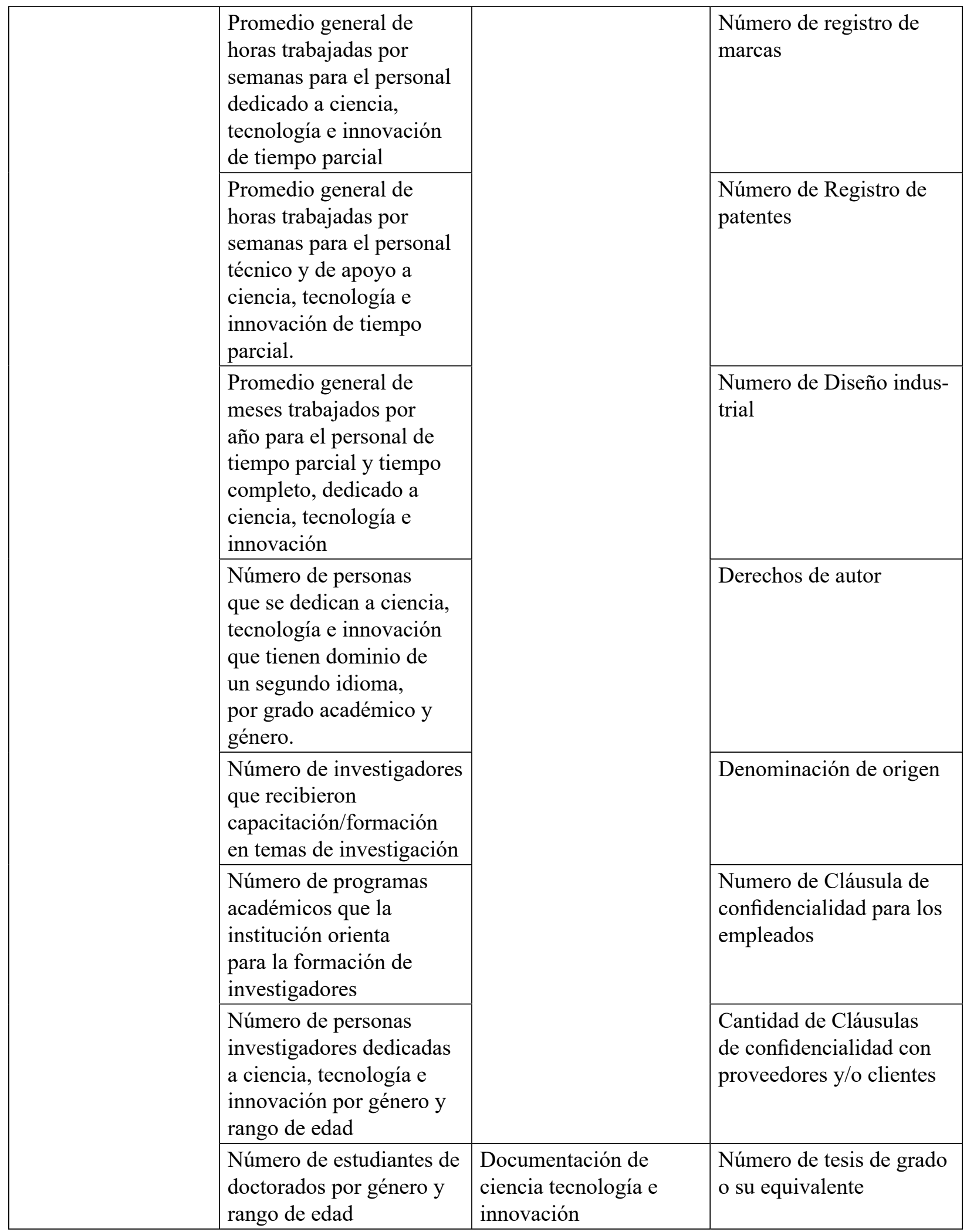




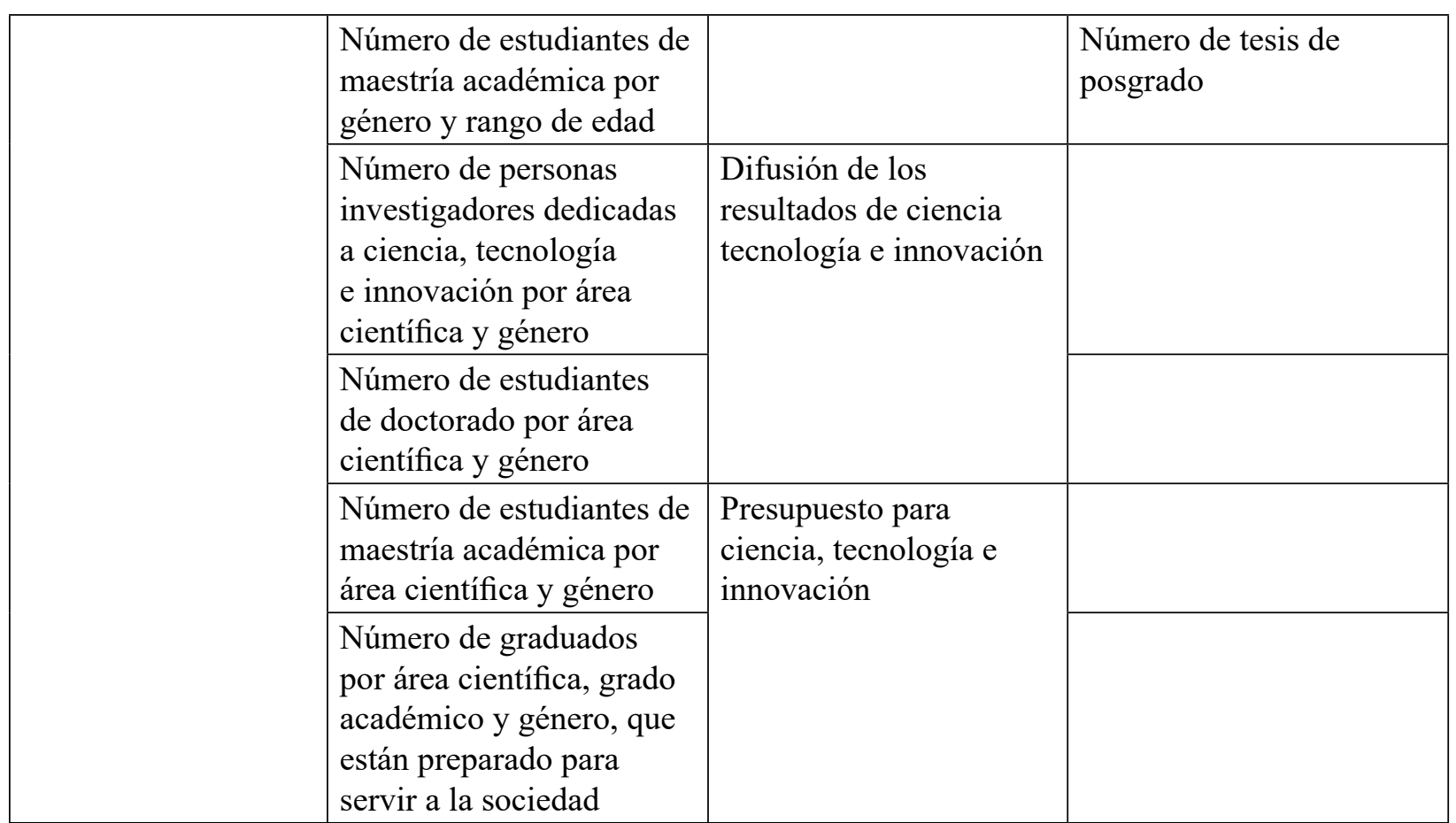

\section{CONCLUSIONES}

La nueva organización institucional de la investigación científica de la UNAN-Managua, implementada en el año 2014, facilitó el cambio hacia una nueva cultura institucional de la UNANManagua de integración de los procesos de investigación-innovación y emprendimiento, lo cual ha tenido impactos positivos que han fortalecido la propia institucionalidad de la universidad. Esto le ha permitido desarrollar exitosamente en el período 2011-2016, los procesos institucionales de CTI-ES en la UNAN-Managua.

La UNAN-Managua ha sentado las bases de una innovación organizacional a través de la DIRINVES, para facilitar el establecimiento y control de los indicadores de ciencia, tecnología e innovación de una forma automatizada usando la plataforma que las TIC brindan hoy en día.

El Modelo I+D+i de la UNAN-Managua, ha permitido la iteración de los procesos de CTIES para facilitar su monitoreo, evaluación, control y mejora continua de tales procesos, por medio de los Sistemas de Información Gerencial de indicadores CTIES.

Es necesario continuar el proceso de nación a nivel del CNU y CONICYT, para desarrollar un software que permita el registro, monitoreo, control y evaluación de los indicadores de CTIES para la UNAN-Managua, estableciendo un mecanismo efectivo y moderno que visibilice la producción científica de Nicaragua. 
Las universidades deben desarrollar una nueva cultura institucional basadas en el registro, monitoreo, control y evaluación de los indicadores de CTIES en sus procesos de investigación, innovación y emprendimiento, para que mediante el desarrollo de nuevas tecnologías los planes de mejora se orienten de manera efectiva a aumentar la productividad, producción, diversificación y valor agregado de los diferentes bienes y servicios en el país, mejorando así el nivel y calidad de vida de la familia nicaragüense.

\section{BIBLIOGRAFÍA}

Alemán Zeledón, F., Lezama Gaitán, L., Gómez, M., \& García, A. (2014). Construcción del Sistema de Indicadores de Ciencia, Tecnología e Innovación en Nicaragua.

Muñoz, A. M. (22 de Mayo de 2011). Gestión de la Innovación, Teoría Proceso y Práctica. Bogota, Bogota, Colombia.

Pedroza, M. E. (2015). Las Etapas del Modelo I+D+i de la UNAN-Managua. En M. E. Pedroza Pacheco, El modelo de gestión de la investigación, Modelo $I+D+i$ de la UNAN-Managua (págs. 28-30). Managua: Editorial Universitaria.

Pedroza, M. E. (2017). Mensaje de Celebración Primer Aniversario del Programa de Doctorado en Gestion de la Calidad de la Investigación Cientifica 2017. Managua: Editorial Universitaria UNAN-Managua.

UNAN-Managua. (2015). Función Investigación. En UNAN-Managua, Plan Estratégico Institucional 2015-2019 (págs. 26-29). Managua: Editorial Universitaria de la UNANManagua.

UNAN-Managua, Dirección de Investigación. (2014). Catalogos de Servicios. En A. d. Emprendimiento, Propuesta de una Oficina de Incubación de Empresas en UNAN Managua (págs. 4-5). Managua: Editorial Universitaria UNAN-Managua.

UNAN-Managua. Comisión de Investigación e Innovación. (2015). TÍTULO DOS: ESTRUCTURA Y FUNCIONAMIENTO. En U.-M. C. Innovación, Reglamento de Investigación e Innovación de las Facultades y el POLISAL (págs. 4-8). Managua: Editorial Universitaria de la UNAN-Managua.

UNAN-Managua. Comisión Facultativa POLISAL. (2015). Capitulo I.Naturaleza, Fines y Objetivos. En U.-M. C. POLISAL, Reglamento de Invesvestigación e Innovación de las Facultades y el Instituto Politecnico de la Salud (págs. 1-5). Managua: Editorial Universitaria de la UNAN-Mangua.

UNAN-Managua. Dirección de Extensión Universitaria. (2012). Sección III Emprendimiento e Innovación. En U.-M. D. Universitaria, Politica de Extensión Universitaria (págs. 13-14). Managua: Editorial Universitaria de la UNAN-Managua.

UNAN-Managua. Dirección de Investigación. (2015). Bases Generales de las Convocatorias. En U.-M. D. Investigación, Normativa para la selección y ejecución de proyectos de innovación de la UNAN-Managua (págs. 3-5). Managua: Editorial Universitaria de la UNAN-Managua.

UNAN-Managua. Dirección de Investigación. (2015). Logros de la Dirección de Investigación (DIRINVES) 2015. Managua: Editorial Universitaria de la UNAN-Managua.

UNAN-Managua. Dirección de Investigación. (2016). Informe de la JUDC 2016, de la UNANManagua. Managua: Editorial Universitaria de la UNAN-Managua. 
UNAN-Managua. Dirección de Investigación. (2014). Propuesta de una Oficina de Incubación de Empresas en UNAN - Managua. Managua: Editorial Universitaria UNAN - Managua.

UNAN-Managua. Dirección de Planificación y Evaluación Institucional. (2015). Introducción. En UNAN-Managua, Plan Estratégico Institucional 2015-2019 (págs. 1-2). Managua: Editorial Universitaria de la UNAN-Managua. 